\title{
Was ist ein menschlicher Embryo?
}

\author{
Vom Orientierungsverlust der Natur in der reproduktiven \\ Biotechnologie $^{1}$
}

Heike Baranzke

\section{Menschliche Embryonen und Modelle mensch- licher Embryonen}

Am 13. Dezember 2018 forderte eine holländisch-US-amerikanische Forschergruppe in der Zeitschrift Nature zu einer internationalen ethischen Diskussion über die Verwendung von Embryo-Modellen aus humanen Stammzellen auf. ${ }^{2}$ Hintergrund ist, dass Entwicklungsbiologie und Stammzellforschung in den letzten Jahren mit einer Vielzahl von Methoden überraschen, mit deren Hilfe embryoähnliche Funktionsäquivalente erzeugt werden können, die im Säugetierbereich auch bereits zu lebendem und fortpflanzungsfähigem Nachwuchs geführt haben. Eine weltweite Klonierungsdebatte löste vor mehr als zwei Jahrzehnten die Geburt des Klonschafs Dolly aus, das nicht aus derVereinigung zweier Keimzellen, sondern durch den Transfer eines somatischen Zellkerns in eine entkernte Eizelle (SCNT-Verfahren) erzeugt wurde. ${ }^{3}$ In den letzten Jahren wurden z.B. durch Verfahren einer tetraploiden Komplementierung lebende Mäuse erzeugt. ${ }^{4}$ Dabei zeigte sich, dass embryonale Stammzellen für sich alleine zwar lediglich pluripotent sind, in Aggregation mit

\footnotetext{
1 Die vorliegenden Ausführungen knüpfen an einen früheren Beitrag an: Heike Baranzke, Der menschliche Embryo - Naturzweck oder Handlungszweck? Eine Kritik an Totipotenz und Potentialitätsargument in der Embryonenschutzdiskussion, in: Entwicklungsbiologische Totipotenz in Ethik und Recht, hg. v. Thomas Heinemann/ Hans-Georg Dederer/Tobias Cantz, Göttingen 2015, 165-222.

2 Nicolas Rivron/Martin Pera and colleagues, Debate ethics of embryo models from stem cells. International discussion must guide research, in: Nature 564 (2018), 183 185.

3 Für die Diskussion exemplarisch Johann S. Ach (Hg.), Hello Dolly? Über das Klonen, Gerd Brudermüller, Christa Runtenberg, Frankfurt a.M. 1998.

${ }^{4}$ Vgl. für die Diskussion Susan Sgodda, Das Kriterium der Totipotenz aus naturwissenschaftlicher Perspektive, in: Entwicklungsbiologische Totipotenz in Ethik und Recht, hg. v. Heinemann/Dederer/Cantz, 13-55, hier: 26-29.
} 
anderen Zellen aber durchaus zur Bildung ganzer Lebewesen stimuliert werden können. Hans Werner Denker warnt seit Jahren eindringlich davor, dass diese Verfahren auch auf Menschen anwendbar seien. ${ }^{5}$ Spontane embryoähnliche Gestaltbildungsprozesse auch von humanen Stammzellkulturen in vitro zeigen sich weiterentwickelbar. Entwicklungsbiologische Grundlagenforschung, die Verbesserung menschlicher Fruchtbarkeit oder gar die Züchtung körpereigener Ersatzorgane erscheinen als lebenswissenschaftliche Verheissungen in einer nicht allzu fernen Zukunft. ${ }^{6}$

Die Forscher sprechen von menschlichen "Embryo-Modellen" und suggerieren damit das Festhalten an einem ontologischen Unterschied zwischen rechten Embryonen als sexuellen Fortpflanzungsprodukten und jenen, auf anderem Wege erzeugten Funktionsäquivalenten ${ }^{7}$, die aber aufgrund ihrer embryonalen Gestaltähnlichkeit und ihres In-vitro-Verhaltens auch die Frage nach der Erlaubtheit menschlicher Embryonenforschung erneut aufzuwerfen scheinen. Kurz gesagt: Das in vitro-Gebilde sieht aus wie ein früher menschlicher Embryo, es verhält sich wie ein früher menschlicher Embryo, aber ist es auch ein menschlicher Embryo? Muss man also solchen embryoähnlichen Gebilden, sofern sie von menschlichen Zellen stammen, denselben Schutz gewähren wie menschlichen Befruchtungsembryonen und sie nach Möglichkeit zur Geburt bringen? Welche Eigenschaft verleiht humanen Zellverbänden den moralischen Status eines menschlichen Embryos? Gestaltähnlichkeit? Ontogenetische Entwicklungsphasen? Sexuelle Produktion aus Gameten? Kann die Beantwortung ethischer Fragen von Naturwissenschaftlern überhaupt erwartet werden? Welche Rolle können Verweise auf natürliche Prozesse hier spielen? Inwiefern kann die Erzeugung von embryonalen Funktionsäquivalenten überhaupt unnatürlich genannt werden, da sie auf biologische Substrate zurückgreift und sich unter Anwendung von Naturgesetzen vollzieht?

In dem vorliegenden Beitrag wird zunächst mit Hilfe einer kritischen Begriffsgeschichte beleuchtet, warum uns diese Forschungsentwicklungen in fundamentale Orientierungsprobleme und Wertkonflikte stürzen. Es gilt, am Beispiel derVerhältnisbestimmung

\footnotetext{
5 Hans Werner Denker, Potentiality of embryonic stem cells: an ethical problem even with alternativ stem cell sources, in: Journal for Medical Ethics 32 (2006), 665-671.

6 Rivron/Pera, Debate ethics of embryo models, 184.

7 Für die hier nicht weiter bedachte moderne Differenzierung von "natürlichen Funktionen" und "künstlichen Zwecken" vgl. den Beitrag von Hans Werner Ingensiep, Entwicklung als "Epigenetische Landschaft". Eine biophilosophische Analyse der Metaphern und Quasiteleologie in der Entwicklungsbiologie, in diesem Band.
} 
von Embryo, Potenzialität und Totipotenz zu realisieren, dass und wie die lebenswissenschaftlich-technischen Erfolge althergebrachte Natürlichkeitsvorstellungen in Frage stellen. Erst wenn dieser Orientierungsverlust begriffen ist, kann die Frage, woran wir uns denn zukünftig orientieren wollen und sollen und welche Rolle die Natur dabei spielt oder spielen soll, neu gestellt werden.

\section{Potenzielle Orientierung? - Von Aristoteles zu den life sciences}

Wer unbekannte Gegenden bereist, ist dankbar, Wegweiser vorzufinden. Sie geben Orientierung darüber, in welcher Richtung die angestrebten Ziele zu finden sind, von denen wir uns dieVerwirklichung eines guten Lebens mit einiger Plausibilität versprechen. Biologische Begriffe wie Same, Frucht oder Gesundheit und Art (species), aber eben auch Embryo, dienen uns seit langem als sprachliche Wegweiser im Zusammenhang mit mannigfaltigen Naturphänomenen. Sie orientieren durch die Erfahrung, welchem Zweck etwas normalerweise dient oder welches Stadium auf dem Weg zum Endziel einer arttypischen Entwicklung erreicht ist. Es sind teleologische Begriffe,

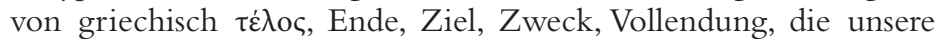
alltäglichen Normalitätserwartungen ausdrücken.

Aristoteles hat diese Orientierungsfunktion der Begriffe zu einer regelrechten teleologischen "Lebenswissenschaft« ausgebaut. Es ist nützlich, sie zu erinnern, da kaum mehr bewusst ist, wie sehr sie unser abendländisches Weltverständnis sowohl in biologischer als auch in kultureller Hinsicht bis heute prägt. Für Aristoteles war die Welt ein Kosmos, eine schöne und gute Ordnung, in der der Mensch eine definierte Stellung innehatte. Vor seinen Augen ordneten sich die Dinge der Natur in zwei Wegweiser-Hinsichten: ${ }^{8}$ Zum einen in eine vertikale Achse, entlang der die Dinge der Natur gemäss zunehmender Vollkommenheit in einer hierarchischen scala naturae aufeinander folgen und der gemäss die unvollkommeneren sniedrigeren

\footnotetext{
8 Im Anschluss an Konrad Gaisers philologische Beobachtungen über "Das zweifache Telos bei Aristoteles" (Naturphilosophie bei Aristoteles und Theophrast., hg. v. Ingemar Düring, Heidelberg 1969, 97-113) unterscheidet Hans Werner Ingensiep zwischen horizontaler und vertikaler Teleologie und veranschaulicht die doppelte Teleologie in einem Achsendiagramm (vgl. das Kapitel über Aristoteles in Hans Werner Ingensiep, Die Geschichte der Pflanzenseele. Philosophische und biologische Entwürfe von der Antike bis zur Gegenwart, Stuttgart 2001, 47), an das sich die vorliegende Darstellung anlehnt.
} 
den vollkommeneren shöheren Wesen zu dienen hatten. ${ }^{9}$ Diese althergebrachte hierarchische Naturordnungsvorstellung äusserer Zweckdienlichkeit zwischen den Dingen der Natur hat Aristoteles in der Politik (1256b) aufgegriffen. Demnach scheint es evident, dass die Pflanzen svon Natur aus für die Tiere da sind, Pflanzen und Tiere aber letztlich dazu bestimmt seien, dem Menschen zu dienen, grundlegend z.B. in der Ernährung. ${ }^{10}$ Aristoteles ergänzt und vertieft diese traditionelle äussere Zweckhierarchie durch seine Lehre von aufeinander aufbauenden artspezifischen Seelenvermögen als innerer Organisation der äusseren Stufenordnung der Lebewesen. So wird die Stellung innerhalb der hierarchischen Kette der Wesen bestimmt von der Anzahl artspezifischer innerer Entwicklungsvermögen, deren zeitlich geordneter, normaler Wachstums- und Reifungsprozess vom Keim bis zum fortpflanzungsfähigen adulten Wesen auf einer horizontalen Achse abgetragen werden kann. Die

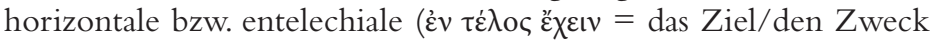
seiner Entwicklung in sich haben) Achse gibt folglich Auskunft auf die Frage, welche Zielgestalt eine biologische Entität aufgrund ihrer inneren artspezifischen Entwicklungsvermögen im Falle günstiger äusserer Entfaltungsbedingungen idealiter verwirklichen kann. Sie darf also quasi ontogenetisch gelesen werden, nicht zuletzt angesichts der Tatsache, dass Aristoteles selbst systematisch embryologische Studien am Hühnerei betrieben hat. ${ }^{11}$

Im Fall pflanzlicher und tierischer Embryonen wird die Vollendungsgestalt im sich fortpflanzenden adulten Organismus erreicht. Im Kontext des Streits über den moralischen Status des menschlichen Embryos wird zu wenig beachtet, dass Aristoteles im Fall des Menschen nicht die Fortpflanzungs-, sondern die Vernunftvermö-

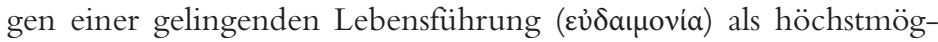
liche Vollendung in der Ethik thematisiert. Die Seelenvermögen des Menschen weisen folglich über eine biologische Bestimmung hinaus, auch wenn sie aus dieser hervorgehen. Sie bilden jedoch die für alle Menschen normale natürliche Grundlage, zu der sich zu verhalten die Menschen tugendethisch herausgefordert sind. Insofern

\footnotetext{
9 Zur wirkmächtigen Ideengeschichte der scala naturae vgl. Arthur O. Lovejoy, Die Kette der Wesen. Geschichte eines Gedankens [1933], übers. v. Dieter Turck, Frankfurt a.M. 1985.

${ }^{10}$ Hans Werner Ingensiep, Heike Baranzke, Natur essen, in: Naturphilosophie, hg. v. Thomas Kirchhoff/Nicole C. Karafyllis u.a., Tübingen 2017, 271-280.

${ }^{11}$ Vgl. Änne Bäumer-Schleinkofer, Die Geschichte der beobachtenden Embryologie. Die Hühnchenentwicklung als Studienobjekt über zwei Jahrtausende, Frankfurt/M. 1993.
} 
weiss auch schon Aristoteles zwischen natürlicher Normalität und tugendethischer Normativität zu unterscheiden.

\section{eudaimonía}

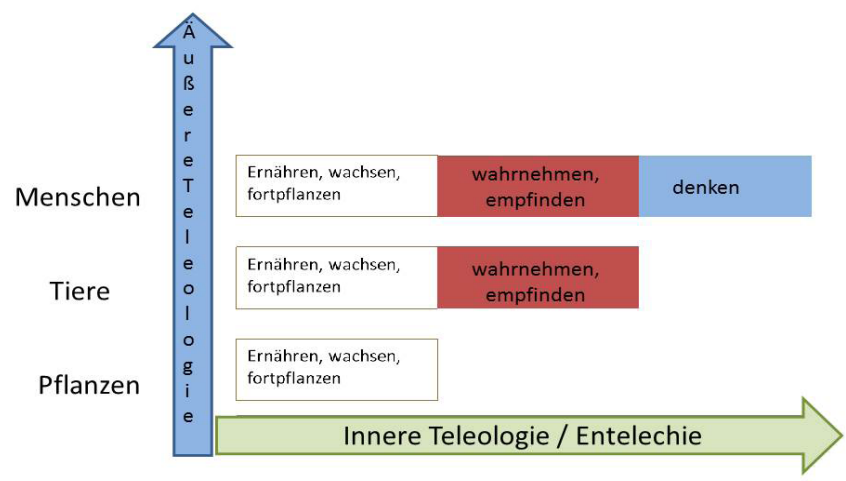

Abb.1: Das doppelte Telos in der aristotelischen Philosophie ${ }^{12}$

Intrinsische Vermögen und ihre Verwirklichung, latinisiert: Potenzi-

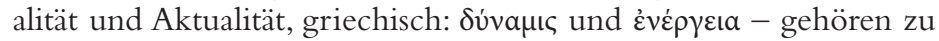
dem terminologischen Handwerkszeug, mit dem Aristoteles natürliche Veränderungsprozesse zu begreifen versucht. Dabei dachte sich Aristoteles die sich stetig selbst verändernde Natur als ein Zwecke setzendes Handlungssubjekt in Analogie zu der Selbsterfahrung eines praktischen, intentionalen Akteurs. Anders ausgedrückt: Die aristotelische Potenzialität - z.B. eines Embryos - ist ein naturteleologischer Begriff, d.h. er interpretiert natürliche Veränderungsprozesse intentional, d.h. handlungstheoretisch. Aristoteles stellt sich die Natur als eine Zwecke setzende Akteurin vor, um Naturprozesse zu begreifen. Er betrachtet speziesspezifische biologische Entwicklungsprozesse nach dem Modell eines bewusst Zwecke setzenden Handwerkers, der ein Artefakt nach seiner Vorstellung gestaltet. Die Natur selbst scheint wie ein TechnikerVeränderungen, z.B. Organbildungs- oder Wachstumsprozesse in lebendigen Entitäten wie z.B. Embryonen, absichtsvoll hervorzubringen.

Kennzeichen der modernen life sciences ist es nun, sich nicht mehr mit der Perspektive einer Akteursanalogie auf die Natur zufrieden $\mathrm{zu}$ geben. Naturerkenntnis ist nicht länger definiert durch die interpretierende Betrachtung von Naturprozessen in vivo. Diese

\footnotetext{
${ }_{12}$ Modifiziert nach Ingensiep, Geschichte der Pflanzenseele, 47.
} 
aristotelisch-kontemplative Naturwissenschaft gleicht einer Vermessung und Kartierung von Naturerfahrungen, die relativ stabile natürliche Zwischenzustände wie Wegweiser zu lesen lernt, indem sie alltägliche Normalitätserwartungen auf Begriffe bringt. Im experimentellen Wissenschaftsparadigma der Neuzeit wird hingegen danach gesucht, durch Kontrolle der Bedingungen im Labor - in vitro - beliebige Naturprozesse experimentell gesteuert hervorzubringen. Wird aber der Ablauf eines Naturprozesses bis zur Verwirklichung seines natürlichen Endzustandes nicht nur - wie bei Aristoteles handlungstheoretisch interpretiert, sondern vom Experimentator durch Kontrolle der Entwicklungsfaktoren auch real hervorgerufen, mutieren Naturprozesse samt ihren fixierbaren Zuständen zu technischen Produkten, zu Artefakten. Welcher dieser produzierten $\mathrm{Zu}-$ stände als vollkommene Endgestalt, als $\tau \dot{\lambda} \lambda$ oc, gelten soll, bestimmen Intentionen und Geschick der Experimentatoren. Die Natur wird ent-teleologisiert, d.h. sie wird in den life sciences nicht länger als ein zweckmässig handelndes Subjekt respektiert, sondern als Stoff für beliebige menschliche Zwecksetzungen gehandhabt. In der Form manipulierbarer Artefakte aber büssen die Dinge der Natur ihren universalen Orientierungscharakter ein. Die schon bei Aristoteles vorliegende Unterscheidung von naturgegebener Normalität (Entelechie) und moralischer Normativität (Eudaimonie) radikalisiert sich bereits seit den Anfängen der Mechanisierung der Natur im 17. Jh. zu einer unüberbrückbar erscheinenden Kluft zwischen Sein und Sollen. Die neuzeitliche experimentelle Naturwissenschaft entfremdet den Menschen folglich von über Jahrtausende gewachsenen Erfahrungen einer Beheimatung in einer unmanipulierten, allen Menschen gleichermassen vorgegebenen natürlichen Seinsordnung. Der Kosmos degeneriert zum naturgesetzlichen Zusammenhang. Zweck und Gestalt ehemaliger Naturdinge als Stadien von Naturprozessen werden unter dem Einfluss lebenswissenschaftlich-technischer Gestaltungsmöglichkeiten gesellschaftspolitische Verhandlungssache - auch im Fall des menschlichen Embryos.

\section{Was ist ein Embryo und wozu? Orientierungs- verluste der Natürlichkeit}

Der Begriff "Embryo" als die aus einem anderen Körper hervorsprossende "Leibesfrucht « (gr. тò ž $\mu \beta \rho v o v=$ die ungeborene Frucht im Mutterleib; auch: neugeborenes Lamm; $\beta \rho v \dot{\omega}=$ hervorsprossen) entstammt in der Antike der vorwissenschaftlichen Alltagsbeobach- 
tung über vorgeburtliches Leben insbesondere im Bereich der Säugetiere und der menschlichen Selbsterfahrung. Die hippokratische Ärzteschule und vor allem Aristoteles, der mit seiner Schrift »De generatione animalium" als der Begründer der Embryologie gilt, stellten »die Zeugungslehre dann erstmals auf eine empirische Basis" am Modell der Hühnchenentwicklung, da die Entwicklung im Hühnerei dem mikroskopisch unbewaffneten Auge zugänglich ist. ${ }^{13}$ Die Vorstellungen über vorgeburtliche Entwicklung sind bis heute dem stetigen Wandel der Theoriebildung unterworfen. Aristoteles deutete seine embryologischen Beobachtungen unter dem Primat seiner teleologischen Lehre von den Vermögen einer artspezifischen Seele als dem entelechialen Lebensbewegungsprinzip im Bereich des Organischen. In der frühen Neuzeit beeinflussten vor allem die Anatomie sowie die Erfindung des Mikroskops, seit dem 19. Jh. die Entdeckung der weiblichen Eizelle durch Karl Ernst von Baer, die Schwann-Schleidensche Zytologie und die Evolutionsbiologie die Vorstellungen von der Gestaltentwicklung des Embryos. Im Zentrum der neuzeitlichen Embryogenese stand die Frage, ob die adulte Endgestalt eines Lebewesens sich bei jedem Individuum nach und nach neu bildet (Epigenesistheorie) oder ob sie schon im Samen vorgebildet sei und lediglich einem Grössenwachstum unterliege (Präformationstheorie). Die grundsätzliche ontologische Frage aber, was ein Embryo ist, war noch um 1900 so unproblematisch, dass sie zu stellen Entwicklungsbiologen erst gar nicht in den Sinn kam. Der Terminus "Embryo « bezeichnete die unhinterfragte Anfangsphase eines Lebewesens vor seiner adulten Gestaltbildung. Erst im Verlauf des 20.Jh.s löst sich das selbstverständliche Vorverständnis von einem Embryo unter dem Einfluss der »Transformation der klassischen Embryologie zur molekularen Entwicklungsbiologie« auf, in deren Kontext es auch zur Entwicklung der In-vitro-Techniken gekommen ist. ${ }^{14}$ Neuartige Klonierungsverfahren haben uns vor Augen

\footnotetext{
${ }_{13}^{13}$ Bäumer-Schleinkofer, Geschichte der beobachtenden Embryologie, 1 u. 9-25.

${ }^{14}$ Johannes Seidel, Schon Mensch oder noch nicht? Zum ontologischen Status humanbiologischer Keime, Stuttgart 2010, 115: Diese Transformation vollzieht sich durch "die Integration vormals unabhängig voneinander arbeitender biologischer Disziplinen«, die Seidel (ebd.) idealtypisch in den folgenden drei Stationen zusammenfasst:

- "Zusammenführung von klassischer Genetik und Biochemie zur molekularen Genetik"

-»Zusammenführung von klassischer Zytologie und molekularer Genetik zur molekularen Zellbiologie»

- "Zusammenführung von klassischer Embryologie und molekularer Zellbiologie zur molekularen Entwicklungsbiologie»
} 
geführt, dass die Erzeugung selbst eines Säugetieres nicht auf die sexuelle Vermischung der Genome zweier Keimzellen angewiesen ist. Die Entdeckung neuer spezifischer Transkriptionsfaktoren in der Stammzellforschung ermöglicht, die Richtung zellulärer Differenzierungsprozesse umzukehren und straft die lebensweltliche Überzeugung von der Irreversibilität von biologischen Entwicklungsprozessen Lügen. Das zunehmende Verständnis genetischer Regulationsvorgänge ermöglicht kontrollierte Gestaltbildungs- bzw. Gestaltveränderungsprozesse. Die Erzeugung zusätzlicher Gliedmassen ist durchaus realisierbar.

Während die bislang aufgezählten Optionen die entelechiale Orientierungsachse einer durch zeitlich aufeinanderfolgende Phasen strukturierten Morphogenese im aristotelischen Diagramm in Frage stellen, lässt die Möglichkeit der Herstellung von InterspeziesChimären auch die vertikale Achse der äusseren teleologischen Stufenordnung distinkter Arten zusammenbrechen. - Ergänzt werden können noch die Überlegungen, die Erzeugung von Lebewesen auf eine andere Materialbasis, nämlich Silizium anstelle von Kohlenstoff, zu stellen. Angesichts dieser neuesten lebenswissenschaftlichen Errungenschaften sind jegliche Orientierung suchenden Normalitätsoder Natürlichkeitsintuitionen angezählt.

Lässt sich aber ein Embryo - ungeachtet seines stofflichen Ursprungs - nicht doch als eine Entität verstehen, aus der ein ganzes Lebewesen entstehen kann? Kann man nicht, wie der deutsche Gesetzgeber zuletzt im Stammzellgesetz versucht hat, einen menschlichen Embryo definieren als eine "jede menschliche totipotente Zelle, die sich bei Vorliegen der dafür erforderlichen weiteren Voraussetzungen $\mathrm{zu}$ teilen und $\mathrm{zu}$ einem Individuum $\mathrm{zu}$ entwickeln vermag« ( $(\mathbb{3}, 4 \mathrm{StZG})$ ? Ist mit der Eigenschaft der Totipotenz nicht doch noch ein unverrückbarer natürlicher Anker gefunden, der erlaubt, zweifelsfrei zu bestimmen, was ein Embryo ist, um darauf aufbauend dann auch menschliche Embryonen identifizieren und unter Entwicklungsschutz stellen zu können?

\section{Totipotenz - Embryo - Potenzialität: virulente Verhältnisbestimmungen}

Die Frage, ob und welche Entitäten totipotent sind, scheint sich auf den ersten Blick an dem natürlichen Entwicklungspotenzial eines Embryos zu orientieren. Aufschlussreich ist, dass der Begriff Totipotenz der experimentellen Entwicklungsbiologie um 1900 entstammt 
und keine im vorwissenschaftlich-lebensweltlichen Sprachgebrauch oder im Recht zuvor verankerte Begriffsgeschichte aufweist. Es handelt sich also um einen neuen biologischen Fachterminus, der just in der Zeit der "Vernaturwissenschaftlichung der Biologie ${ }^{15}$ geprägt wird, die auch in der Embryologie zu einer Transformation von einer beobachtenden zu einer experimentell arbeitenden Disziplin führt. ${ }^{16}$ Dennoch wurde Totipotenz nicht als Definition eines Embryos eingeführt. ${ }^{17}$

Der Term "Totipotenz" wurde ursprünglich zur experimentellen Exploration von Gestaltbildungsprozessen (Morphogenese) in frühen Organismen eingeführt. Diese Prozesse liessen sich am besten am Beispiel solcher tierischer Embryonen verfolgen, deren Entwicklung extrauterin beobachtbar ist. Daher wurden die entwicklungsbiologischen Experimente vor allem an Seeigeln und Amphibien durchgefuihrt. Man wollte herausfinden, ob schon der frühe befruchtete Keim den fertigen ’präformierten` Bauplan für die Endgestalt des adulten Organismus miniaturhaft enthält oder ob die Organismusgestalt erst durch sukzessiv eingeleitete Regulationsprozesse - "epigenetisch « - allmählich entsteht. Dazu isolierte der von der vitalistischen Idee eines Ganzheitsfaktors inspirierte Entwicklungsbiologe Hans Driesch Blastomeren durch Schütteln aus frühen Seeigelkeimen, während sein mechanistisch denkender Kollege Wilhelm Roux eine Blastomere in amphibischen Zweizellembryonen durch Anstechen mit einer heissen Nadel abtötete und zu weniger befriedigenden Ergebnissen kam. Die klassische Definition in dem ersten, von Roux verfassten Fachwörterbuch der Entwicklungsbiologie vergleicht Totipotenz als das Gestaltungsvermögen eines Teils

\footnotetext{
${ }^{15}$ Ebd., 111. Hans Werner Ingensiep zeigt am Beispiel der im 20. Jh. verwendeten metaphorischen Visualisierungen der Entwicklungsvorstellungen ("Epigenetische Landschaft« vs. "Flipperautomat») den meist unreflektierten und damit subversiv wirkenden Umschlag von "natürlichen« funktionalen Verläufen zu technisch gesteuerten Prozessen detaillierter auf. Vgl. den Beitrag von Hans Werner Ingensiep, Entwicklung als "Epigenetische Landschaft«. Eine biophilosophische Analyse der Metaphern und Quasiteleologie in der Entwicklungsbiologie, in diesem Band.

${ }^{16}$ Ebd., 111f. Seidel spricht von der »Transformation der Embryologie von einer deskriptiven zu einer experimentell arbeitenden Disziplin«. Da aber auch die experimentelle Wissenschaft deskriptiv und nicht etwa präskriptiv im moralischen Sinne ist, ist der weniger missverständliche Gegenbegriff zu sexperimentell $>$ beobachtend .

${ }^{17}$ So Christian Kummer, Zweifel an der Totipotenz. Zur Diskussion eines vom deutschen Embryonenschutz überforderten Begriffs, in: Stimmen der Zeit 222 (2004), 459-472; auch Johannes Huber, Totipotenz - überfordertes Kriterium der Schutzwürdigkeit? Eine naturphilosophische Untersuchung zu den biologischen Grundlagen eines normativ gewordenen Begriffs, Berlin 2009.
} 
einer befruchteten Eizelle mit dem Vermögen einer ganzen befruchteten Eizelle, »ein ganzes Lebewesen zu entwickeln «18.

Driesch und Roux reflektierten jedoch nicht, dass erst ihre Manipulation das Regenerationsvermögen der bereits differenzierten Keimteile entfacht hatte und die Totipotenz ihres Keimteils folglich ein Artefakt war. Fachwissenschaftlich uneindeutig ist bis heute, von welchen Entitäten Totipotenz definitionsgemäss ausgesagt werden könne. Während in der Stammzellforschung Totipotenz als Eigenschaft von isolierten Einzelzellen betrachtet wird, ${ }^{19}$ bezeichnet der Term in der Embryologie auch die Regulationsfähigkeit eines embryonalen Zellverbandes zu einer Organogenese und Entwicklung zu einem Individuum, nachdem seine Gesamtzellzahl experimentell reduziert wurde. ${ }^{20}$ Roux' klassische Totipotenzdefinition scheint zumindest terminologisch für beide Verwendungsweisen offen zu sein, insofern die (isolierte) "Furchungszelle" nur als Beispiel eines "erst sehr wenig specificierten< Keimteiles « dient. ${ }^{21}$ Mitte des 20. Jh.s wurden die u.a. von Hans Spemann vorgedachten ersten Zellkerntransplantationsversuche durchgeführt, "um festzustellen, ob sich eine 'Anlages, in einem fremden 'Wirt sherkunftsgemäss', also unabhängig von ihrer neuen Umgebung, entwickelt und ob sie ihrerseits ihre neue Umgebung beeinflusst «. ${ }^{22}$ Mit dieser Entwicklung des Somatic Cell Nuclear Transfer (SCNT)-Verfahrens, der Transplantation eines somatischen Zellkerns in eine entkernte Eizelle, die durch die Geburt des ersten SCNT-Schafs als "DollyMethode " bekannt wurde, wurde dann sogar von der Totipotenz von Zellkernen gesprochen. Diese Redeweise wird heute allerdings aufgrund der Entdeckung des massgeblichen Anteils des Eizellplasmas an der Entwicklungsfähigkeit kritisiert. ${ }^{23}$ Die späteren Reprogrammierungstechniken in der Stammzellforschung haben jedoch

\footnotetext{
18 Wilhelm Roux, Terminologie der Entwicklungsmechanik der Tiere und Pflanzen, Leipzig 1912, 409f.

${ }_{19}$ So bei Sgodda, Kriterium der Totipotenz, die versucht, den Totipotenzbegriff noch strikt aus der spezifischen Stammzellforschungsperspektive für Einzelzellen zu reservieren, aber zugibt, dass "(a)ngesichts dieser Entwicklungsfähigkeit zu einem ganzen Organismus aus einem artifiziell kombinierten Zellgebilde (scil.: der tetraploiden Komplementierung, HB) [...] die Einführung eines adäquaten Terminus ähnlich dem Totipotenzbegriff möglicherweise angebracht« ist (ebd, 29; vgl. auch ebd., 41).

${ }^{20}$ Henning M. Beier, Totipotenz und Pluripotenz. Von der klassischen Embryologie zu neuen Therapiestrategien, in: Stammzellforschung und therapeutisches Klonen, hg. v. Fuat S. Oduncu/Ulrich Schroth/Wilhelm Vossenkuhl, Göttingen 2002, 36-54.

${ }^{21}$ Roux, Terminologie, 409.

22 Bäumer-Schleinkofer, Geschichte der beobachtenden Embryologie, 248.

${ }^{23}$ Vgl. Sgodda, Kriterium der Totipotenz, 24; Huber, Totipotenz, 98-100.
} 
gezeigt, dass Forscher für die Entwicklung ganzer Lebewesen nicht einmal mehr auf natürliche Eizellderivate angewiesen sind. ${ }^{24}$ Somit wird deutlich, dass Totipotenz der faktische Ex-post-Nachweis einer erfolgreichen Organismusbildung aus einer individuellen biologischen Entität ist, die um dieses Nachweises willen technisch isoliert und kontrolliert werden muss. Totipotenz ist folglich ein experimenteller, labortechnischer Aktualitäts-, kein natürlicher Potenzialitätsbegriff aristotelischer Prägung. Denn Aristoteles interpretierte lediglich die Normalitätserwartung (Potentialität) aus alltäglichen Fortpflanzungserfahrungen mit Menschen und Tieren in Analogie zu einem Zwecke setzenden technischen Akteur. Moderne Lebenswissenschaftler bestimmen selbst die Zwecke des Material gewordenen, entsubjektivierten Natursubstrats, um Normalitätserwartungen zu verändern und menschliche Handlungsmöglichkeiten zu erweitern. Totipotenz ist folglich ein Experimentalterm, dem die Erwartung innewohnt, Lebewesen auf bislang ungekannten Wegen erzeugen zu können.

\section{5. ... und was ist ein »menschlicher Embryo«? - Die Frage nach dem Menschen zwischen Poten- zialitätsargument und Speziesismusvorwurf}

Dass Totipotenz kein notwendiges Definitionskriterium für (menschliche) Embryonen ist, erhellt die Tatsache, dass wir - z.B. im Kontext der assistierten Reproduktion - auch solche Befruchtungsprodukte als Embryonen ansprechen, die nicht entwicklungsfähig sind. Wir sprechen dann z.B. von ’kranken Embryonen. Es stellt sich aber

\footnotetext{
${ }^{24}$ Für die Möglichkeiten der »Erzeugung von Gameten aus pluripotenten Stammzellen" vgl. Tobias Cantz, in: Entwicklungsbiologische Totipotenz in Ethik und Recht, hg. v. Heinemann/Dederer/Cantz, 57-66. - R. Sparrow (In vitro eugenics, in: Journal for Medical Ethics (JME) 2013,1-7; doi 10.1136/medethics-2012-101200) berichtet, dass bereits lebende Mäuse geboren sind, die aus Gameten erzeugt wurden, die zuvor aus ES-Zellen entwickelt worden waren (ebd., 2) und beschreibt Zukunftsszenarien, die sich aus den Kombinationsmöglichkeiten der verschiedenen Techniken ergeben: "If it proves possible to derive gametes from iPS cells, or from embryonic stem cells derived from embryos created by (hypothetical) somatic cell nuclear transfer (SCNT), this would allow the creation of the genetic offspring of any person from whom a somatic cell containing nuclear DNA could be sourced. Thus, in vitro gametogenesis could serve as a powerful new technology to overcome infertility, especially for men who are unable to produce viable sperm, women who have undergone premature menopause, and for those who have lost their gonads due to injury or had them removed in the course of cancer treatment." (ebd., 3)
} 
angesichts der erstaunlichen entwicklungsbiologischen Forschungsergebnisse die Frage, ob Totipotenz ein hinreichendes Kriterium ist, um auch alle jene konstruierten zellbiologischen Funktionsäquivalente, von denen zumindest prinzipiell der Nachweis technisch assistierter Ganzheitsbildungsfähigkeit im Tierreich erbracht worden ist, Embryonen genannt werden sollen. So ist jedenfalls der deutsche Gesetzgeber im Embryonenschutzgesetz und im Stammzellgesetz verfahren. Der Medizinrechtler Jochen Taupitz kritisiert den gesetzgeberischen Sprachgebrauch als schwerwiegenden Wertungswiderspruch, da es offensichtlich menschliche Embryonen gebe, die möglichst vollzählig aus der Petrischale in den Uterus einer Frau transferiert werden sollen, und andere, bei denen der Transfer unter Strafandrohung verboten sei..$^{25}$

Dieser unterstellte Wertungswiderspruch ergibt sich für Taupitz aus der Zuschreibung der traditionellen Bezeichnung "Embryo « zu entwicklungsfähigen biologischen Entitäten, die nicht aus einer sexuellen Gametenvereinigung hervorgegangen sind. Die traditionelle Bindung des Embryoseins an Befruchtungsembryonen erweckt den Eindruck, als ob noch scharf zwischen einer naturnahen Reproduktionsmedizin und einer artifiziellen Stammzellforschung unterschieden werden könnte. ${ }^{26}$ Der Experimentalterm Totipotenz führt aber klar vor Augen, dass wir Menschen bereits jetzt über ontogenetische Alternativen verfügen, die für höhere Tiere - jüngst erstmals das SCNT-Verfahren für die Gattung der Primaten ${ }^{27}$ - längst verwirklicht worden sind. In der Forschung werden auch für den Humanbereich nicht nur vielfältige medizinische Visionen, sondern

${ }^{25}$ Vgl. Jürgen Taupitz, Lebensbeginn und Lebensschutz aus dem Blickwinkel des deutschen Rechts, in: Lebensbeginn im Spiegel des Medizinrechts. Beiträge der 2. Tagung der Medizinrechtslehrerinnen und Medizinrechtslehrer 2010 in Zürich, hg. v. Brigitte Tag, Baden-Baden 2011, 33-48.

${ }^{26}$ Ein erster "Wertungswiderspruch" wurde vor »Dolly« u.a. in Deutschland im Kontext der Novellierung der Schwangerschaftskonfliktgesetzgebung diagnostiziert. Unter Abstrahierung von Frauen als Grundrechtsträgerinnen wurden nicht selten von Seiten der Befürworter einer Liberalisierung verbrauchender Embryonenforschung Befruchtungsembryonen in utero und in vitro verglichen und bemängelt, dass letztere durch das Embryonenschutzgesetz konsequenter geschützt seien als durch eine liberalisierte Schwangerschaftskonfliktgesetzgebung. Der Unterschied zu der späteren Wertungswiderspruchsthese besteht darin, dass es nun nicht mehr um die Gegenüberstellung von Befruchtungsembryonen in vivo und in vitro geht, sondern um mehr oder weniger snatürliche und artifizielle menschliche In-vitro-Embryonen.

${ }^{27}$ Die ersten Klonaffen nach der "Dolly«-Methode: Zhong-Zhong und Hua Hua, in: Ärzteblatt v. 24. Jan. 2018, https://www.aerzteblatt.de/nachrichten/88711/ Die-ersten-Klonaffen-nach-der-Dolly-Methode-Zhong-Zhong-und-Hua-Hua (30.12.2018). 
auch schon die Möglichkeiten biologisch eigenen Nachwuchses für Singles und homosexuelle Paare ohne heterosexuelle Befruchtungsvorgänge entworfen.

Es lohnt noch einmal ein Blick zurück auf die doppelte Teleologie des Aristoteles, um präziser zu bestimmen, worin genau die neuartigen moralischen Herausforderungen der modernen experimentellen Biotechnologie im Vergleich zu der vormodernen, weitgehend kontemplativen Naturforschung liegen. Sie bestehen darin, dass die fundamentale technische Kontrollierbarkeit und Veränderbarkeit von Naturprozessen uns aufnötigen $z u$ entscheiden, wieviel Orientierungskraft wir technisch unmanipulierten normalen Naturprozessen noch aus guten Gründen zugestehen wollen. Das Ideal unberührter, gedeihender Natur, symbolisiert auf der entelechialen Achse, orientiert seit der Antike naturrechtliche Argumentationen in Philosophie und Theologie. Aber auch Krankheiten und Degenerationsprozesse sind natürlich. Sie lassen sich eventuell aus der ökologischen Perspektive als zweckvoll im Ganzen der biotischen Natur darstellen. Aus der Perspektive individueller, glückssuchender Lebewesen sind sie aber schwerlich als biologische Vollendungszwecke darzustellen, um mit den biotechnologischen Verheissungen überzeugend zu konkurrieren. Der Preis für diese biotechnologische Utopie ist jedoch die zunehmende Einbusse von naturbedingten Normalitätserwartungen bezüglich fundamentaler Lebensprozesse mit der unausweichlichen Folge, unsere biologischen Eckdaten in immer grösserem Masse gesellschaftlich aushandeln zu müssen, wie es an den Grenzen des menschlichen Lebens ohnehin schon geschieht..$^{28}$ Dann aber wirft uns die wachsende Naturbeherrschung mit ihrer radikalen Infragestellung entelechialer Normalitätsorientierungen, die für sich allerdings noch keine normativen Rechtfertigungen darstellen, noch unvermittelter als schon bei Aristoteles auf die anthropologisch-ethische und politische Frage zurück, wie wir

\footnotetext{
${ }^{28}$ Unverhandelbar ist hingegen der vorpositive Menschenwürde- und Menschenrechtsstatus geborener Menschen, ganz unabhängig davon, mit welchen biotechnischen Mitteln sie auch immer ins Dasein gebracht wurden. Das hat die öffentliche Gegenrede gegen Sibylle Lewitscharoffs Vortrag über die moderne Reproduktionsmedizin im Dresdner Schauspielhaus am 2. März 2014 in ihrem Vortrag "Von der Machbarkeit. Die wissenschaftliche Bestimmung über Geburt und Tod" noch einmal unzweifelhaft klargestellt (vgl. z.B. Büchner-Preisträgerin hält Skandalrede zu künstlicher Befruchtung, Spiegel-online v. 6.3.2014, http://www.spiegel.de/kultur/ literatur/lewitscharoff-rede-buechner-preistraegerin-zu-befruchtung-und-onanie-a-957254.html (31.12.2018). - Es ist aber notwendig darüber zu diskutieren, welche Formen technischer Manipulationen am menschlichen Lebensbeginn möglicherweise den Charakter von Menschenrechtsverletzungen aufweisen.
} 
uns als Menschen begreifen wollen und sollen und wie wir uns

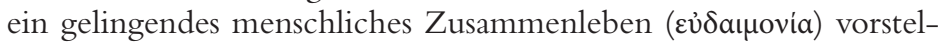
len. Wenn wir diese Frage wiederum rein biologisch beantworten - wie im Rahmen der Diskussion des Potenzialitätsarguments oft geschieht - und Menschsein auf die Zugehörigkeit zur biologischen Spezies homo sapiens sapiens reduzieren, ${ }^{29}$ dann mündet die Naturbeherrschung in eine szientistische Technokratie, denn normative Menschenwürde- und Menschenrechtsideen sind biologisch nicht definierbar.

- Dr. Heike Baranzke ist Dozentin für Theologische Ethik an der Bergischen Universität Wuppertal und hat in diversen interdisziplinären Forschungsprojekten mitgearbeitet. Zu ihren Forschungsschwerpunkten gehören Facetten einer Ethik der Menschenwürde in unterschiedlichen Bereichen der Bio- und Gesundheitsethik, Kantische Ethik sowie interdisziplinäre Grundlagenreflexionen.

${ }^{29}$ Vgl. dazu ausführlicher Baranzke, Der menschliche Embryo, bes. 197-208. 